\title{
Bilinear Mappings and the Frame Operator
}

\author{
Enrico Au-Yeung \\ Pacific Institute for the Mathematical Sciences, Vancouver, Canada \\ Email: enricoauy@math.ubc.ca
}

Received March 28, 2013; revised April 30, 2013; accepted May 26, 2013

Copyright (C) 2013 Enrico Au-Yeung. This is an open access article distributed under the Creative Commons Attribution License, which permits unrestricted use, distribution, and reproduction in any medium, provided the original work is properly cited.

\begin{abstract}
The theory of frames has been actively developed by many authors over the past two decades, both for its applications to signal processing, and for its deep connections to other areas of mathematics such as operator theory. Central to the study of frames is the frame operator. We initiate an investigation that extends the frame operator to the bilinear setting.
\end{abstract}

Keywords: Harmonic Analysis; Frames

\section{Introduction}

The theory of frames was initiated by Duffin and Schaeffer [1] to study some deep problems in non-harmonic Fourier series. For more than three decades, their ideas did not seem to generate much interest outside of nonharmonic Fourier series. Finally in 1986, Daubechies, Grossman, and Meyer [2] in their groundbreaking paper observed that frames can be used for painless nonorthogonal expansions for functions. Since then, frames have been used in signal processing, image processing, and data compression, as well as being studied for their deep connections to operator theory [3]. Frames are important in signal processing because they can be used to provide stable reconstruction of signals. For background in the theory of frames, see [4-6]. Central to the study of frames is the frame operator.

We initiate an investigation that extends the frame operator to the bilinear setting. Bilinear operators in harmonic analysis have been studied by many authors, see, for example, [7-10]. The conjecture that the bilinear Hilbert transform can be extended to a bounded operator has remained open for some 30 years before it was settled in the celebrated work of Lacey and Thiele [11]. The results in our current work extend the results concerning a class of bilinear operators known as paraproducts; these operators are better behaved than the usual products of functions, see [12]. The results in this article indicate that there is a rich underlying theory that awaits to be developed. The present work only touches on certain aspects of that theory.

Let $H$ be a separable Hilbert space. A sequence $\left\{x_{n}\right\}_{n=1}^{\infty}$ of elements in $H$ is a frame for $H$ if there exist positive constants $A$ and $B$ such that

$$
\forall f \in H, \quad A\|f\|^{2} \leq \sum_{n=1}^{\infty}\left|\left\langle f, x_{n}\right\rangle\right|^{2} \leq B\|f\|^{2} .
$$

For the rest of this article, the Hilbert space $H$ is taken to be $L^{2}\left(\mathbb{R}^{d}\right)$. Let $\mathcal{S}\left(\mathbb{R}^{d}\right)$ be the Schwartz space of rapidly decreasing smooth functions on $\mathbb{R}^{d}$. The Fourier transform of a function $f \in L^{2}\left(\mathbb{R}^{d}\right)$ is defined by $\hat{f}(\zeta)=\int_{\mathbb{R}^{d}} f(x) \mathrm{e}^{-2 \pi i \zeta \cdot x} \mathrm{~d} x$.

\section{Main Results}

We begin with a useful lemma that will simplify our calculations later.

Lemma 2.1 (Convolution with a radial function is a self-adjoint operator)

Let $\psi \in \mathcal{S}\left(\mathbb{R}^{d}\right)$ be a radial function, i.e. $\forall x \in \mathbb{R}^{d}$, $\psi(x)=\overline{\psi(|x|)}$.

Define an operator $T: L^{2}\left(\mathbb{R}^{d}\right) \rightarrow L^{2}\left(\mathbb{R}^{d}\right)$ by

$$
\begin{aligned}
& \forall f \in L^{2}\left(\mathbb{R}^{d}\right), \\
& (T f)(x)=\int_{\mathbb{R}^{d}} f(x-y) \psi(x) \mathrm{d} y=(f * \psi)(x) .
\end{aligned}
$$

Then $T$ is a self-adjoint operator. That means,

$$
\forall f, g \in L^{2}\left(\mathbb{R}^{d}\right),\langle T f, g\rangle=\langle f, T g\rangle .
$$

Proof. Let $f, g \in L^{2}\left(\mathbb{R}^{d}\right)$.

We first note that $T f \in L^{2}\left(\mathbb{R}^{d}\right)$ since

$\|f * \psi\|_{2} \leq\|f\|_{2}\|\psi\|_{1}$. 


$$
\begin{aligned}
\langle T f, g\rangle & =\int_{\mathbb{R}^{d}}\left(\int_{\mathbb{R}^{d}} f(x-y) \psi(x) \mathrm{d} y\right) \overline{g(x)} \mathrm{d} x \\
& =\int_{\mathbb{R}^{d}}\left(\int_{\mathbb{R}^{d}} f(y) \psi(x-y) \mathrm{d} y \overline{g(x)} \mathrm{d} x\right. \\
& =\int_{\mathbb{R}^{d}}\left(\int_{\mathbb{R}^{d}} f(y) \overline{\psi(y-x)} \mathrm{d} y\right) \overline{g(x)} \mathrm{d} x, \quad \psi(x)=\overline{\psi(|x|)} \\
& =\int_{\mathbb{R}^{d}} \int_{\mathbb{R}^{d}} f(y) \overline{\psi(y-(x+y))} \overline{g(x+y)} \mathrm{d} x \mathrm{~d} y, \quad x \mapsto x+y \\
& =\int_{\mathbb{R}^{d}} \int_{\mathbb{R}^{d}} f(y) \overline{\psi(-x)} \overline{g(x+y)} \mathrm{d} x \mathrm{~d} y \\
& =\int_{\mathbb{R}^{d}} \int_{\mathbb{R}^{d}} f(y) \overline{\psi(x)} \overline{g(y-x)} \mathrm{d} x \mathrm{~d} y, \quad x \mapsto-x \\
& =\int_{\mathbb{R}^{d}} f(y) \int_{\mathbb{R}^{d}} \overline{\psi(x)} \overline{g(y-x)} \mathrm{d} x \mathrm{~d} y \\
& =\int_{\mathbb{R}^{d}} f(y) \overline{(g * \psi)(y)} \mathrm{d} y \\
& =\langle f, T g\rangle .
\end{aligned}
$$

Consider a bilinear operator $B: H \times H \rightarrow H$.

$\forall f, g \in H, B(f, g) \in H$, and $B$ is linear in each of the two variables separately.

Let $\psi, \phi \in \mathcal{S}\left(\mathbb{R}^{d}\right)$ with compact support, and

$$
\int_{\mathbb{R}^{d}} \psi(x) \mathrm{d} x=0, \quad \int_{\mathbb{R}^{d}} \phi(x) \mathrm{d} x=1 . \quad \psi \text { is radial, i.e. } \psi(x)=\overline{\psi(|x|)} .
$$

We normalize $\psi$ so that $\int_{0}^{\infty}\left|\hat{\psi}(t \zeta)^{2}\right| \frac{\mathrm{d} t}{t}=1$. Write $\psi_{t}(x)=\frac{1}{t^{d}} \psi\left(\frac{x}{t}\right)$.

Define $B(f, g)$ by:

$$
B(f, g)(x)=\int_{0}^{\infty} \psi_{t} *\left(\left(\psi_{t} * f\right) \cdot\left(\phi_{t} * g\right)\right)(x) \frac{\mathrm{d} t}{t}
$$

Consider $\left\langle B(f, g), e_{n}\right\rangle=\int_{\mathbb{R}^{d}} \int_{0}^{\infty} \psi_{t} *\left(\left(\psi_{t} * f\right) \cdot\left(\phi_{t} * g\right)\right) \cdot e_{n} \frac{\mathrm{d} t}{t} \mathrm{~d} x, \quad$ where $e_{n} \in H$.

$$
\begin{aligned}
\left\langle B(f, g), e_{n}\right\rangle & =\int_{\mathbb{R}^{d}} \int_{0}^{\infty} \psi_{t} *\left(\left(\psi_{t} * f\right) \cdot\left(\phi_{t} * g\right)\right) \cdot e_{n} \frac{\mathrm{d} t}{t} \mathrm{~d} x \\
& =\int_{\mathbb{R}^{d}}\left(\psi_{t} * f\right) \cdot\left(\phi_{t} * g\right)(x) \cdot\left(\psi_{t} * e_{n}\right)(x) \mathrm{d} x, \quad \text { by Lemma } 2.1 \\
& =\int_{\mathbb{R}^{d}}\left(\psi_{t} * f\right) \cdot\left(\left(\phi_{t} * g\right)(x) \cdot\left(\psi_{t} * e_{n}\right)(x)\right) \mathrm{d} x \\
& =\int_{\mathbb{R}^{d}} f(x) \cdot \psi *\left(\left(\phi_{t} * g\right) \cdot\left(\psi_{t} * e_{n}\right)\right)(x) \mathrm{d} x,
\end{aligned}
$$

where the last line follows from

$\langle f, T h\rangle=\langle T f, h\rangle$, with $h=\left(\psi_{t} * e_{n}\right) \cdot\left(\phi_{t} * g\right)$.

We are now ready to construct the frame operator. Let $\left\{e_{n}\right\}_{n=1}^{\infty}$ be a sequence in $H$.

$\left\langle M^{*} c, f\right\rangle_{H}=\langle c, M f\rangle_{l_{2}}=\sum_{n} c_{n}\left\langle B(f, g), e_{n}\right\rangle_{H}$ by

Fix $g \in H$. Define the analysis operator $\mathrm{M}: \mathrm{H} \rightarrow \mathrm{l}_{2}$

$\forall f \in H$,

$M f=\left(\left\langle B(f, g), e_{1}\right\rangle,\left\langle B(f, g), e_{2}\right\rangle,\left\langle B(f, g), e_{3}\right\rangle, \cdots\right)$.

Using $S=M^{*} M$, we obtain

$$
\begin{aligned}
S f & =M^{*} M f \\
& =M^{*}\left(\left\langle B(f, g), e_{1}\right\rangle,\left\langle B(f, g), e_{2}\right\rangle,\left\langle B(f, g), e_{3}\right\rangle, \cdots\right)
\end{aligned}
$$

The frame operator $S: H \rightarrow H$ is given by $S=M^{*} M$.

First, calculate the adjoint operator of $\mathrm{M} . \mathrm{M}: \mathrm{H} \rightarrow \mathrm{l}_{2}$. $M^{*}: l_{2} \rightarrow H$.

Let $c=\left(c_{1}, c_{2}, c_{3}, \cdots,\right)$ be a sequence in $l_{2}$. Then,

By the above calculations on $B(f, g)$, we see that:

$$
\begin{aligned}
& \left\langle B(f, g), e_{n}\right\rangle \\
= & \int_{\mathbb{R}^{d}} \int_{0}^{\infty} f(x) \cdot\left(\psi_{t} *\left(\left(\phi_{t} * g\right) \cdot\left(\psi_{t} * e_{n}\right)\right)\right)(x) \frac{\mathrm{d} t}{t} \mathrm{~d} x \\
= & \int_{\mathbb{R}^{d}} f(x) B\left(e_{n}, g\right)(x) \mathrm{d} x,
\end{aligned}
$$


where

$$
B\left(e_{n}, g\right)(x)=\int_{0}^{\infty} \psi_{t} *\left(\left(\psi_{t} * e_{n}\right) \cdot\left(\phi_{t} * g\right)\right)(x) \frac{\mathrm{d} t}{t} .
$$

Therefore,

$$
\begin{aligned}
& \left\langle M^{*} c, f\right\rangle_{H}=\langle c, M f\rangle_{l_{2}} \\
& =\sum_{n=1}^{\infty} c_{n} \cdot\left\langle B\left(e_{n}, g\right), f\right\rangle \\
& =\left\langle\sum_{n=1}^{\infty} c_{n} \cdot\left\langle B\left(e_{n}, g\right), f\right\rangle, \text { where } B\left(e_{n}, g\right) \in H\right.
\end{aligned}
$$

So, $M^{*} c=\sum_{n=1}^{\infty} c_{n} \cdot B\left(e_{n}, g\right)$,

$c=\left(c_{1}, c_{2}, c_{3}, \cdots\right), M^{*}: l_{2} \rightarrow H$.

Hence, $S f=\sum_{n=1}^{\infty}\left(\left\langle B(f, g), e_{n}\right\rangle_{H} \cdot B\left(e_{n}, g\right)\right), S: H \rightarrow H$.

i.e. $S f=\sum_{n=1}^{\infty}\left\langle f, B\left(e_{n}, g\right)\right\rangle_{H} \cdot B\left(e_{n}, g\right)$. This is our frame operator.

We have constructed a frame operator with a bilinear mapping. Let us summarize all our calculations in the following lemma.

Lemma 2.2 (Frame operator with bilinear mapping) Let $H$ be the Hilbert space $L^{2}\left(\mathbb{R}^{d}\right)$.

Let $\psi, \phi \in \mathcal{S}\left(\mathbb{R}^{d}\right)$ with compact support, and $\int_{\mathbb{R}^{d}} \psi(x) \mathrm{d} x=0, \quad \int_{\mathbb{R}^{d}} \phi(x) \mathrm{d} x=1 . \quad \psi \quad$ is radial, i.e.

$\psi(x)=\overline{\psi(|x|)}$

We normalize $\psi$ so that $\int_{0}^{\infty}\left|\hat{\psi}(t \zeta)^{2}\right| \frac{\mathrm{d} t}{t}=1$. Write $\psi_{t}(x)=\frac{1}{t^{d}} \psi\left(\frac{x}{t}\right)$

Consider a bilinear operator $B: H \times H \rightarrow H$ defined by Equation (1).

Then the frame operator $S: H \rightarrow H$ is given by

$$
\begin{aligned}
S f & =\sum_{n=1}^{\infty}\left\langle B(f, g), e_{n}\right\rangle B\left(e_{n}, g\right) \\
& =\sum_{n=1}^{\infty}\left\langle f, B\left(e_{n}, g\right)\right\rangle B\left(e_{n}, g\right) .
\end{aligned}
$$

Proof. Our calculations established the lemma.

To prove that this bilinear operator is bounded, we need some preparation.

Lemma 2.3 Let $H$ be a separable Hilbert space (or a separable Banach space). Let $M$ be a dense subspace of $H$. Let $B: M \times H \rightarrow H$ be a bilinear operator such that

$$
\forall f \in M, \forall g \in H,\|B(f, g)\|_{H} \leq C \cdot\|f\|_{H}\|g\|_{H} .
$$

Then the above inequality holds for all $f$ in $H$, for all $g$ in $H$, and $B$ extends to a bounded bilinear operator from $H \times H$ to $H$.

Proof. For each $f$ in $H$, there exists a sequence $\left\{f_{n}\right\}_{n=1}^{\infty} \in M$ such that

$\left\|f-f_{n}\right\|_{H} \rightarrow 0$, as $n \rightarrow \infty$. Since for each $g \in H$,

$$
\begin{aligned}
& \left\|B\left(f_{m}, g\right)-B\left(f_{n}, g\right)\right\|_{H} \\
= & \left\|B\left(f_{m}-f_{n}, g\right)\right\|_{H} \\
\leq & C \cdot\left\|f_{m}-f_{n}\right\|_{H} \cdot\|g\|_{H} \quad \text { by }(2)
\end{aligned}
$$

So, for each $g \in H,\left\{B\left(f_{n}, g\right)\right\}_{n=1}^{\infty}$ is a Cauchy sequence in $H$.

Hence, $B\left(f_{n}, g\right)$ converges in $H$ to an element in $H$, and we can define a bounded bilinear operator $B(f, g): H \times H \rightarrow H$ by $B(f, g)=\lim _{n \rightarrow \infty} B\left(f_{n}, g\right)$.

Definition 2.4 (BMO) If $f$ is a locally integrable function in $\mathbb{R}^{d}$, we say that $f \in B M O$ if there exists $a$ constant $A<\infty$, such that for any cube $Q \subseteq \mathbb{R}^{d}$,

$$
\frac{1}{Q} \int_{Q}\left|f(x)-f_{Q}\right| \mathrm{d} x \leq A .
$$

Here, $f_{Q}$ is the average of $f$ over the cube. The integration is over the cube. The smallest bound $A$ for which the above inequality is satisfied is taken to be the norm of $f$, and is denoted by $\|f\|_{B M O}$.

sFor background on BMO functions, see Chapter 4 of [13], as well as the seminal paper by C. Fefferman and Stein [14]. The next theorem on BMO functions, together with Lemma 2.3, will allow us to establish the boundedness of the bilinear operator.

Theorem 2.5 Let $\psi \in \mathcal{S}\left(\mathbb{R}^{d}\right)$ be such that $\int_{\mathbb{R}^{d}} \psi(x) \mathrm{d} x=0$. Let $\phi$ be a bounded, integrable function that is positive, radial, and decreasing. Write $\phi_{t}(x)=\frac{1}{t^{d}} \phi\left(\frac{x}{t}\right)$. Let $b \in B M O$. Then

$$
\begin{aligned}
& \int_{\mathbb{R}^{d}} \int_{0}^{\infty}\left|\phi_{t} * f(x)\right|^{2}\left|\psi_{t} * b(x)\right|^{2} \frac{\mathrm{d} x \mathrm{~d} t}{t} \\
& \leq C\|b\|_{B M O}^{2} \int_{\mathbb{R}^{d}}|f(x)|^{2} \mathrm{~d} x .
\end{aligned}
$$

Definition 2.6 If $f \in B M O \cap L^{2}\left(\mathbb{R}^{d}\right)$, then $f \in L_{B M O}^{2}\left(\mathbb{R}^{d}\right)$.

Remark 2.7 The space $L_{B M O}^{2}\left(\mathbb{R}^{d}\right)$ is a dense subspace of $L^{2}\left(\mathbb{R}^{d}\right)$ in the topology of $L^{2}\left(\mathbb{R}^{d}\right)$.

Theorem 2.8 (Boundedness of the bilinear operator)

Assume the hypothesis of Lemma 2.2. Define $B(f, g)$ by:

$$
\begin{aligned}
& \forall f, g \in L^{2}\left(\mathbb{R}^{d}\right), \\
& B(f, g)(x)=\int_{0}^{\infty} \psi_{t} *\left(\left(\psi_{t} * f\right) \cdot\left(\phi_{t} * g\right)\right)(x) \frac{\mathrm{d} t}{t} .
\end{aligned}
$$

Then there exists $C>0$ such that for each $e_{n} \in H$, 
with $\left\|e_{n}\right\|=1$,

$$
\begin{aligned}
& \forall f \in L_{\text {BMO }}^{2}\left(\mathbb{R}^{d}\right), g \in L^{2}\left(\mathbb{R}^{d}\right), \\
& \left\langle B(f, g), e_{n}\right\rangle \leq C \cdot\|f\|_{2} \cdot\|g\|_{2} .
\end{aligned}
$$

Proof. Let $f \in L_{B M O}^{2}\left(\mathbb{R}^{d}\right)$. Let $g \in L^{2}\left(\mathbb{R}^{d}\right)$. Let $e_{n} \in L^{2}\left(\mathbb{R}^{d}\right)$, with $\left\|e_{n}\right\|_{2}=1$.

$$
\begin{aligned}
\left\langle B(f, g), e_{n}\right\rangle & \\
= & \int_{\mathbb{R}^{d}} \int_{0}^{\infty} \psi_{t} *\left(\left(\psi_{t} * f\right) \cdot\left(\phi_{t} * g\right)\right)(x) \cdot e_{n}(x) \frac{\mathrm{d} t}{t} \mathrm{~d} x \\
= & \int_{\mathbb{R}^{d}} \int_{0}^{\infty}\left(\psi_{t} * f\right) \cdot\left(\phi_{t} * g\right)(x) \cdot\left(\psi_{t} * e_{n}\right)(x) \frac{\mathrm{d} t}{t} \mathrm{~d} x \\
\leq & \left(\int_{\mathbb{R}^{d}} \int_{0}^{\infty}\left|\psi_{t} * f(x)\right|^{2} \cdot\left|\phi_{t} * g(x)\right|^{2} \frac{\mathrm{d} t}{t} \mathrm{~d} x\right)^{1 / 2} \\
& \cdot\left(\int_{\mathbb{R}^{d}} \int_{0}^{\infty}\left|\psi_{t} * e_{n}(x)\right|^{2} \frac{\mathrm{d} t}{t} \mathrm{~d} x\right)^{1 / 2} \\
\equiv & I_{1} \cdot I_{2}
\end{aligned}
$$

Let $G(x)=\int_{0}^{\infty}\left|\phi_{t} * g(x)\right|^{2} \frac{\mathrm{d} t}{t}$. Then $\|G\|_{2}^{2}=C_{1} \cdot\|g\|_{2}^{2}$

by Plancherel Theorem. Hence,

$$
\begin{aligned}
I_{1}^{2} & =\int_{\mathbb{R}^{d}} \int_{0}^{\infty}\left|\psi_{t} * f(x)\right|^{2} \cdot\left|\phi_{t} * g(x)\right|^{2} \frac{\mathrm{d} t}{t} \mathrm{~d} x \\
& \leq C_{1} \cdot\|f\|_{B M O}^{2} \int_{\mathbb{R}^{d}}|g(x)|^{2} \mathrm{~d} x .
\end{aligned}
$$

In the last inequality, we used Theorem 2.5. Another application of Plancherel Theorem gives the following:

$$
I_{2}^{2}=\int_{\mathbb{R}^{d}} \int_{0}^{\infty}\left|\psi_{t} * e_{n}(x)\right|^{2} \frac{\mathrm{d} t}{t} \mathrm{~d} x \leq C_{2} \cdot\left\|e_{n}\right\|_{2} .
$$

Hence,

$$
\left\langle B(f, g), e_{n}\right\rangle \leq C \cdot\|f\|_{\text {ВМО }} \cdot\|g\|_{2} \cdot\left\|e_{n}\right\|_{2} .
$$

Therefore $B$ is a bounded operator on $L_{B M O}^{2}\left(\mathbb{R}^{d}\right) \times L^{2}\left(\mathbb{R}^{d}\right)$. Recall that the space $L_{B M O}^{2}\left(\mathbb{R}^{d}\right)$ is a dense subspace of $L^{2}\left(\mathbb{R}^{d}\right)$. By Lemma $2.3, B$ extends to a bounded operator on $L^{2}\left(\mathbb{R}^{d}\right) \times L^{2}\left(\mathbb{R}^{d}\right)$. This concludes the proof of the theorem.

\section{Acknowledgments}

The author is grateful to Professor John Benedetto from
University of Maryland for teaching him the subject of harmonic analysis and the theory of frames.

\section{REFERENCES}

[1] R. J. Dun and A. C. Schaeer, "A Class of Nonharmonic Fourier Series," Transactions of the American Mathematical Society, Vol. 72, No. 2, 1952, pp. 341-366. doi:10.1090/S0002-9947-1952-0047179-6

[2] A. G. I. Daubechies and Y. Meyer, "Painless Nonorthogonal Expansions," Journal of Mathematical Physics, Vol. 27, 1986, pp. 1271-1283.

[3] D. Han and David Larson, "Frames, Bases and Group Representations," Memoirs of the American Mathematical Society, Vol. 147, 2000, p. 697.

[4] O. Christensen, "An Introduction to Frames and Riesz Bases," Springer-Birkhauser, New York, 2003.

[5] I. Daubechies, "Ten Lectures on Wavelets," SIAM, Philadelphia, 1992.

[6] C. Heil and D. Walnut, "Continuous and Discrete Wavelet Transforms," SIAM Review, Vol. 31, No. 4, 1989, pp. 628-666. doi:10.1137/1031129

[7] R. R. Coifman and Y. Meyer, "On Commutators of Singular Integrals and Bilinear Singular Integrals," Transactions of the American Mathematical Society, Vol. 212, 1975 , pp. $315-331$.

[8] L. Grafakos and N. J. Kalton, "The Marcinkiewicz Multiplier Condition for Bilinear Operators," Studia Mathematica, Vol. 146, 2001, pp. 115-156.

[9] L. Grafakos and R. Torres, "Discrete Decompositions for Bilinear Operators and Almost Diagonal Conditions," Transactions of the American Mathematical Society, Vol. 354, 2002, pp. 1153-1176.

[10] N. Tomita, "A Hormander Type Multiplier Theorem for Multilinear Operators," Journal of Functional Analysis, Vol. 259, 2010, pp. 2028-2044.

[11] M. Lacey and C. Thiele, "On Calderon's Conjecture," Annals of Mathematics, Vol. 149, No. 2, 1999, pp. 475496. doi:10.2307/120971

[12] A. Benyi, D. Maldonado and V. Naibo, "What Is a Paraproduct?" Notices of the American Mathematical Society, Vol. 57, No. 7, 2010, pp. 858-860.

[13] E. M. Stein, "Harmonic Analysis," Princeton University Press, Princeton, 1993.

[14] C. Feerman and E. Stein, "Hp Spaces of Several Variables," Acta Mathematica, Vol. 129, 1972, pp. 137- 193. 